\title{
An Advanced Parametric Thermal Model for High Power LED Modules
}

\author{
Paul Fulmek ${ }^{1}$, Peter Haumer ${ }^{1}$, Franz Peter Wenzl ${ }^{2}$, Wolfgang Nemitz ${ }^{2}$, \\ Roman Beigelbeck ${ }^{3}$, Johann Nicolics ${ }^{1}$ \\ ${ }^{1}$ Department of Applied Electronic Materials, ISAS, TU-Wien, \\ A-1040 Wien, Österreich \\ ${ }^{2}$ Institute for Surface Technologies and Photonics, Joanneum Research \\ Forschungsgesellschaft $\mathrm{mbH}, \mathrm{A}-8160$ Weiz, Österreich \\ ${ }^{3}$ Center for Integrated Sensor Systems, Danube University Krems, \\ A-2700 Wiener Neustadt, Österreich
}

fulmek@tuwien.ac.at

\begin{abstract}
:
This paper presents a comprehensive static and transient thermal simulation model for high-power LED-modules to produce white light with a defined quality. Since high power and high efficiency InGaN-LEDs emit blue light, a color conversion element (CCE) is necessary to produce the white light. The performance of the CCE is dependent on the temperature and on the blue light flux. While a classical goal of thermal simulation and thermal management is to optimise a thermal resistance for a defined heat source, the goal of our simulations is to control the heat dissipation in the LED-chip and in the CCE, which are dependent on each other and on their temperatures. Both components, LED and CCE, exhibit efficiencies that are nonlinearily dependent on the input power (electrical power, light power) and temperature. Accordingly, the nonlinear properties of both, LED and CCE, need to be integrated into the simulation model. Electrical measurement, optical measurement, temperature measurement, and IR-thermography are used to verify the model-setup. The static and transient simulations use the electrical LED current or voltage as input and determine the temperature distributions in the LED and the CCE, thus allowing for a systematic approach to improve the white light quality and the reliability and lifetime of PC-LED-modules under various operation modes.
\end{abstract}

Key words: High-power LED, color conversion elements, thermal FEM simulation, IR thermography, junction temperature estimation.

\section{Introduction}

In the last years, solid state lighting showed an increasingly huge potential as future illumination technology. General lighting applications and automotive lighting already benefit from the high efficiency of LED lighting systems [1][2]. Since high efficiency LEDs emit light in the blue color range, a color conversion element (CCE) is needed to generate the desired white light with a defined quality (color temperature, chromaticity coordinates, color rendering index $\mathrm{CRI}$, etc.). The CCE typically consists of phosphor particles embedded in a silicone matrix (phosphor converted LED) [3]

Typical high-power LEDs with an emitting surface of $\sim 1 \mathrm{~mm}^{2}$ can emit a total blue light flux of $\sim 1 \mathrm{~W}$, and produce a heat flux of several $\mathrm{W} / \mathrm{mm}^{2}$, which heats up the LED and has to be dissipated towards the heat sink. The power, the dominant wavelength, and the spectral bandwidth of the blue light emitted by the LED depends on the forward current and on the LED temperature, especially on the effective temperature of the active region of the LED chip, commonly referred to as junction temperature [4][5][6]. In real high power LEDs with multiple quantum wells (MQW) and a rather large emitting surface, covered partly by an electrode structure, however, there is no single junction nor a unique junction temperature [7][8]. For practical reasons, the effective junction temperature together with the forward LED-current is used to describe the performance of the LED. The concept of a modified Shockley equation allows using the forward voltage of the LED to determine this junction temperature with a resolution better than $1 \mathrm{~K}$ [9]. For our simulations, we assume a (time independent) complete and unique description of the LED operation by the triplet of forward voltage, forward current, and junction 
temperature [10]. These three figures uniquely define the optical output power and spectrum, as well as the loss power, which is responsible for an increase of the junction temperature.

The color conversion element (CCE) represents a second source of heat in this LED-system. Phosphor particles in a silicone matrix absorb blue photons from the LED and convert them with temperature dependent quantum efficiency $\mathrm{QE}$ into photons with a lower wavelength. From the limited quantum efficiency and the Stokes shift we can calculate the local temperature dependent loss power in the CCE. The distribution of the absorption figure in the CCE is calculated by ray-tracing simulations, for a given particle distribution. Due to the relatively low thermal conductivity of the CCE in comparison to the LED-chip, the temperatures in the CCE are much higher than the junction temperature [11][12][13].

Steady state experiments and simulations are used to develop our model and to verify the materials parameters. Transient simulations can be used to evaluate the effect of pulsed LED operation (PWM) on the temperature distribution in the LED and the CCE, to optimise for the white light quality, and the reliability and lifetime of the complete phosphor converted LED-module, under various operation modes.

\section{LED Sample and Model}

In order to develop a comprehensive LEDmodule model, we have chosen a typical InGaN LED chip (Cree EZ900 II) mounted on an insulated metal substrate (IMS). The IMS consists of an $\mathrm{Al}$ sheet with an insulating dielectric layer $(90 \mu \mathrm{m})$ and a $\mathrm{Cu}$ layer $(70 \mu \mathrm{m})$. Samples of LED-modules have been prepared with and without CCE. Figure 1 shows the schematic chip layout from the datasheet [14]. The datasheet contains figures showing typical inter-dependencies of forward voltage, forward current, junction temperature, light flux, and dominant wavelength.
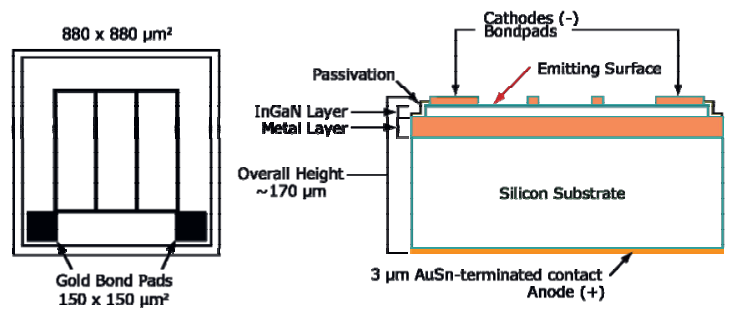

Fig. 1. Geometry of a Cree EZ900 II InGaN-LED (original images from datasheet [14]).

Figure 2 shows a photo of the LED-chip mounted on the IMS by a silver-based conductive adhesive as anode and the wirebonded cathode at the LED top-surface.
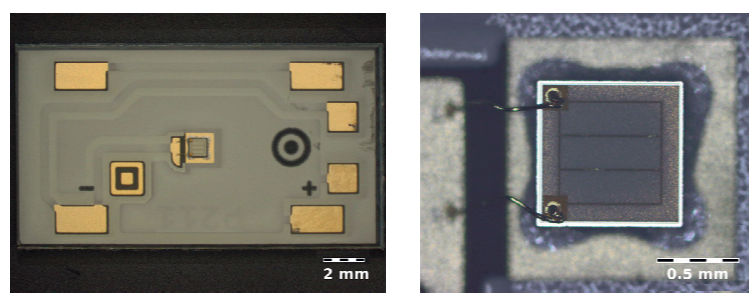

Fig. 2. Sample chip on IMS (18 mm x $10 \mathrm{~mm})$. The bottom anode is mounted using a silver based adhesive and wire-bonds contact the top cathode.

As there is no detailled information about the internal structure of the LED-chip available, we had to design an equivalent geometry model, with equivalent materials parameters. From microsection images, however, we could verify some principle dimensions of the LED chip, our adhesive layers, and the IMS. The simplified 3D-geometry model has been created using the open-source-software Gmsh/GetDP [15][16].

Figure 3 shows the 3D-geometry model. Due to the symmetries we can use only $1 / 8$ of the real sample thus reducing the FEM calculation effort. The complex and unknown multiplequantum-well structure (MQW) of the LED chip has been replaced by a single equivalent block where the LEDs loss-power is dissipated. Additionally, we omitted the influence of the electrode structure of the top-cathode, thus neglecting additional inhomogeneities in the current distribution, light emission, power dissipation, and consequently the temperature distribution [8]. The Si chip carrying the metal layer below the MQW is used as anode and therefore acts as series resistor with additional temperature dependent Joule losses.

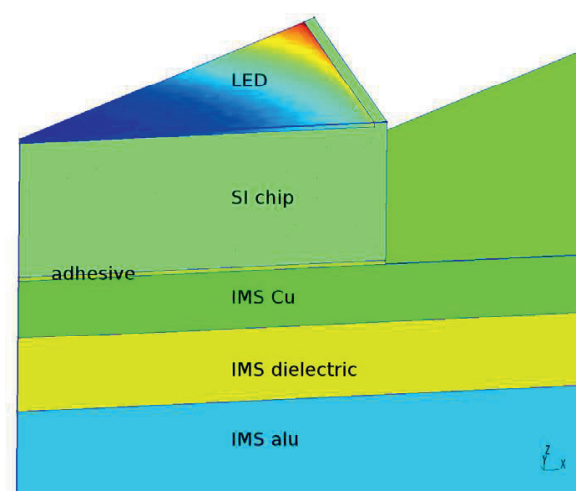

Fig. 3. Model geometry: The active LED layers are modelled as a single thin layer on top of the Si-chip. A typical temperature distribution is shown on the top of the active $L E D$ domain.

Within this simplified sample geometry, the heat equation (1) is solved for temperature $T$, using the open-source GPL package GetDP. 


$$
\rho \cdot c_{p} \cdot \frac{\partial T}{\partial t}-\nabla \cdot(\lambda \nabla T)=\dot{q}_{v}
$$

The materials parameters mass density $\rho$, specific heat capacity $c_{p}$, thermal conductivity $\lambda$ may all appear temperature dependent. The source term $q_{\mathrm{v}}$ represents the volume heat power and is determined by experimental LED characterisation. GetDP uses a Galerkin representation of the heat equation, which contains also surface terms, e.g. for convective and radiative cooling.

\section{Experimental LED Characterisation}

For our simulations, we use the LED forward current as only input parameter, with appropriate boundary conditions for coolertemperature and convective cooling. The respective temperature dependent heat dissipation in the system is determined from characteristic maps and their analytical approximations. These properties of the LED sample are experimentally determined by the pulsed forward voltage method in an integrating Ulbricht sphere [5][6][9]. Within certain limits, we may assume a constant temperature for the whole LED module equal to the junction temperature. This temperature is measured by a Pt100 sensor at the top of the IMS and controlled by a Peltier element attached to the bottom of the IMS. Short rectangular current pulses are applied to the module, the forward voltage and the emitted light flux and spectrum are measured.

The results are characteristic maps (Figure 4 and Figure 5) describing the correlations between forward voltage, forward current, junction temperature, emitted blue light, and consequently the dissipated loss power and the LED efficiency. This loss power acts as heat source in the active LED-region.

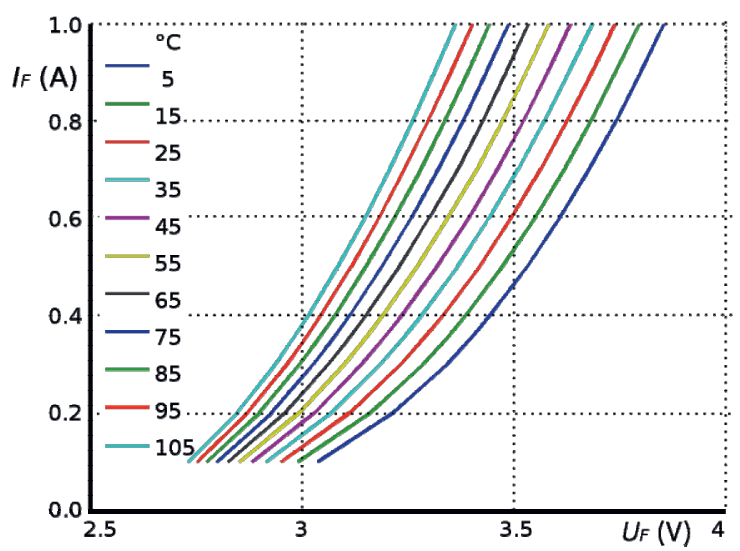

Fig. 4. UIT characteristic map: Forward voltage $U_{F}$, forward current $I_{F}$, junction temperature $T$ determined by the forward voltage method with $30 \mu$ s current pulses.

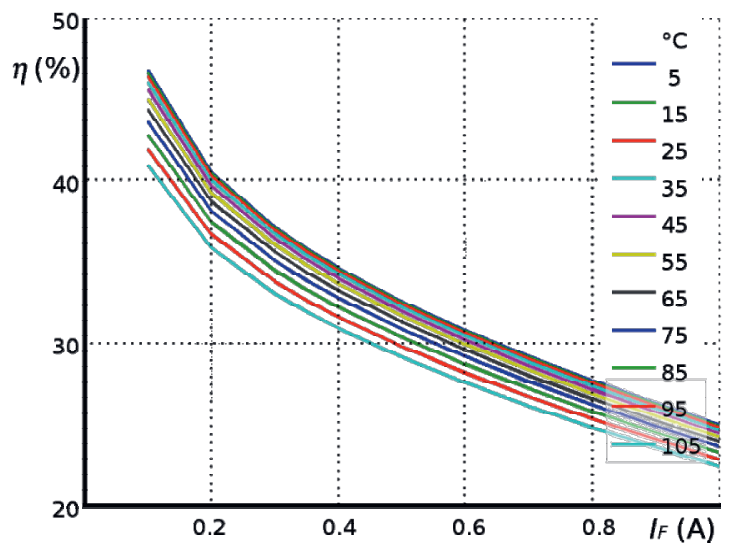

Fig. 5. EIT characteristic map: LED efficiency $\eta=P_{\text {blue }} / P_{\text {el }}$ versus forward current $I_{F}$ and junction temperature $T$.

The non-linear simulation for a chosen forward current iteratively calculates the temperature distribution in the system, the forward voltage, the emitted blue light flux, and consequently the LED loss power.

As the evaluation of the experiments did not show any relevant temperature dependence of the LED series resistor, we could safely neglect its influence on the measured voltage and light flux.

\section{Identification and Verification of Parameters}

Finite element modelling FEM starts with an idealised, simplified geometry model and materials parameter estimated from bulk material data. As neither the geometry-model, nor the available bulk materials data can exactly reflect the real sample properties, the final parameterisation of the FEM-model is the result of an iterative process. The temperature distribution calculated by a first simulation with an initial set of estimated parameters is compared to the (surface) temperature distribution experimentally determined by IRthermography. Figure 6 shows typical emission corrected thermograms taken at a forward current of $350 \mathrm{~mA}$ and $25^{\circ} \mathrm{C}$ cooler temperature with two different thermography systems operating in the 3-5 $\mu \mathrm{m}$ (Infratec IR 8300) and in the $8-12 \mu \mathrm{m}$ (Agema THV900) range of wavelengths.

From a tolerance analysis, we can identify the parameters which have the largest impact on the simulation result, and adapt them accordingly to the measurement data. Table 1 summarises the final thermal materials data-set used in our simulations. The thermal resistance of the adhesive layer showed the most important impact on the simulation result. 

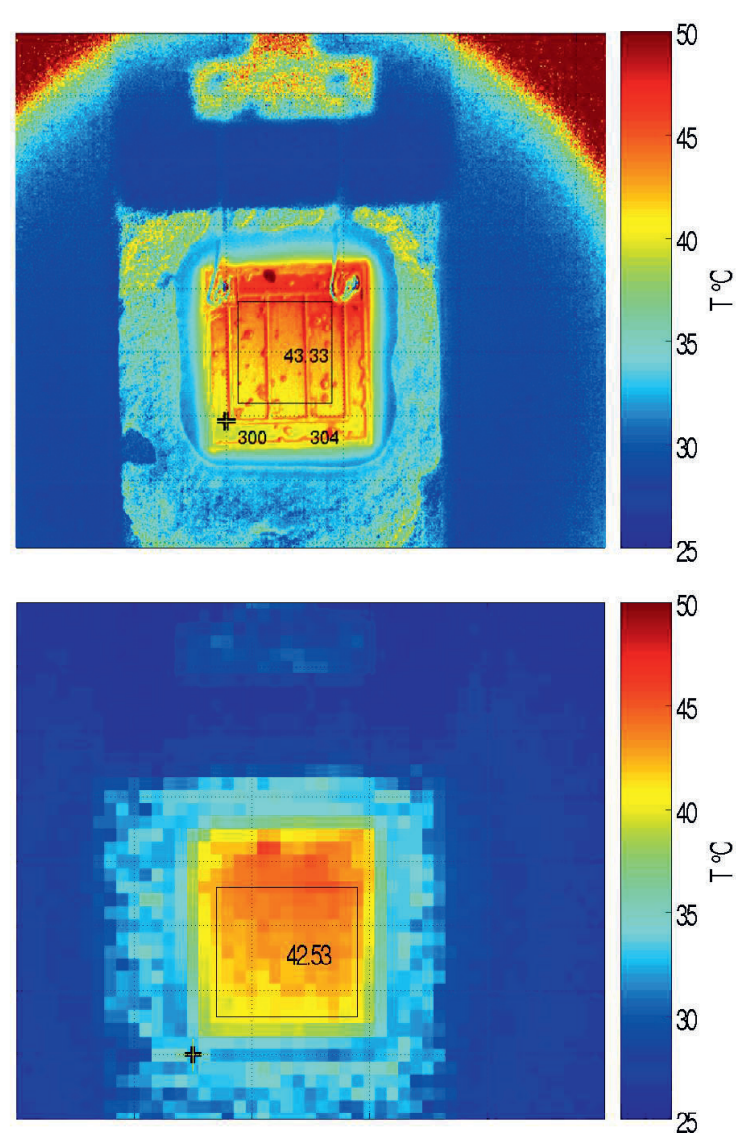

Fig. 6. Emission-corrected thermograms of the LED at $350 \mathrm{~mA}$ and $25^{\circ} \mathrm{C}$ cooler temperature. Top: Infratec IR8300, spatial resolution $\sim 6 \mu \mathrm{m} /$ pixel. Bottom: Agema THV900, spatial resolution $\sim 70 \mu \mathrm{m} /$ pixel.

Tab. 1: Thermal material parameters applied in the FEM model.

\begin{tabular}{|l|c|c|c|}
\hline & $\begin{array}{c}\text { Thermal } \\
\text { conductivity } \\
(\mathrm{W} / \mathrm{mK})\end{array}$ & $\begin{array}{c}\text { Heat } \\
\text { Capacity } \\
(\mathrm{J} / \mathrm{kgK})\end{array}$ & $\begin{array}{c}\text { Specific } \\
\text { density } \\
\left(\mathrm{kg} / \mathrm{m}^{3}\right)\end{array}$ \\
\hline LED & 45 & 325 & 5320 \\
\hline Si-Chip & 100 & 700 & 2340 \\
\hline Adhesive & 0.45 & 800 & 7000 \\
\hline IMS-Cu & 270 & 420 & 8300 \\
\hline IMS-Diel. & 2.60 & 900 & 3000 \\
\hline IMS-AI & 138 & 897 & 2700 \\
\hline
\end{tabular}

\section{LED Simulation}

The simulations for a chosen forward current $I_{F}$ start with an initial value for the temperature distribution in the LED-module. From the characteristic maps we determine the power dissipated in the LED, as well as the power of the emitted blue light. The blue light power $P_{\text {blue }}$ is used as input parameter for the additional colour conversion element simulations. In an iterative loop, the final temperature distribution is determined.

$$
\begin{aligned}
& U_{F}\left(I_{F}, T\right) \Rightarrow \text { Fig.4 } \\
& P_{e l}=I_{F} \cdot U_{F}\left(I_{F}, T\right) \\
& \eta\left(I_{F}, T\right) \Rightarrow \text { Fig.5 } \\
& P_{\text {loss }}=P_{e l} \cdot\left(1-\eta\left(I_{F}, T\right)\right)
\end{aligned}
$$

Figure 3 shows a resulting temperaturedistribution on the LED surface. The representative junction temperature $T$ is calculated as mean temperature across the volume of the active LED-domain. The inhomogeneity of the real temperaturedistribution is neglected.

Figure 7 depicts temperature line-scans across the center of the LED surface and the IMS surface from simulations compared to linescans from emission corrected IRthermography acquired by two different systems: AGEMA THV900 (8-12 $\mu \mathrm{m})$ and Infratec IR8300 (3-5 $\mu \mathrm{m})$. The largest temperature gradient occurs between LED surface and IMS surface, indicating a significant thermal resistance, which has to be attributed mainly to the thermal resistance of the Si-chip and the adhesive layer. Accordingly, we can verify the effective thermal conductivity of the adhesive layer (see Table 1).

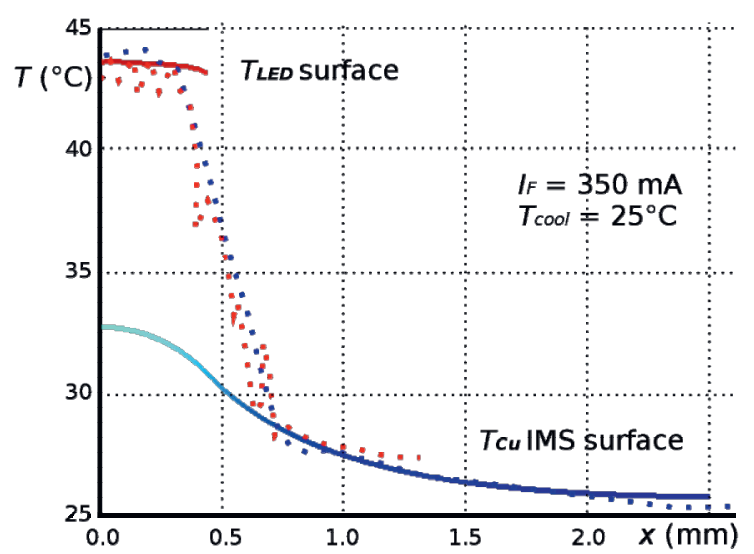

Fig. 7. Comparison of FEM simulation results (solid lines) and results from emission-corrected IRthermography (cmp. Fig. 6) (blue dots: Agema THV900, red dots: Infratec IR8300).

In Figure 8 we collect time dependent simulation results for the temperatures across the vertical center-line through LED and IMS. Within the first $40 \mu \mathrm{s}$, the temperature in the active LED domain raises by less than $0.5 \mathrm{~K}$, while the IMS temperatures remain at the initial value. These results show the temperature error level due to the pulse length for the pulsed 
forward voltage measurements. Accordingly, we can justify a temperature resolution better than $0.5 \mathrm{~K}$ for our experimental characterisation.

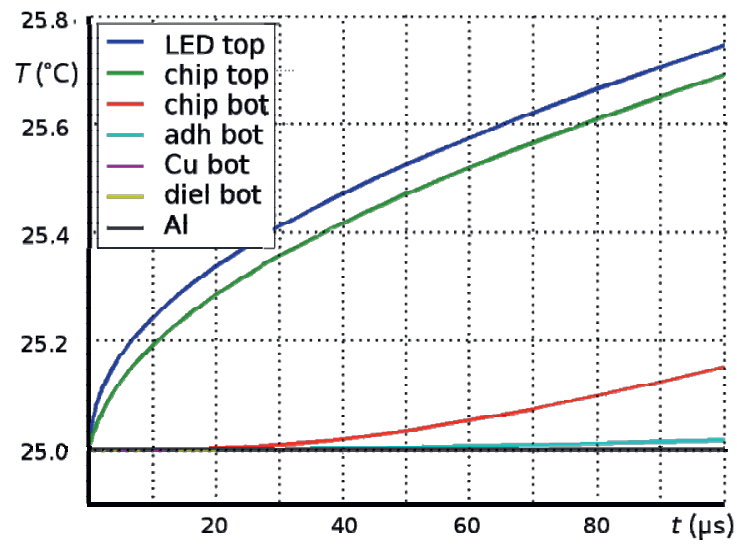

Fig. 8. Transient simulation results at $I_{F}=350 \mathrm{~mA}$. Within the first $40 \mu \mathrm{s}$, the temperature in the active LED domain rises by less than $0.5 \mathrm{~K}$, while the IMS temperature remains cold.

\section{LED Module with CCE}

The most common approach for white light generation relies on a combination of blue LED light and the excited emission from one or more phosphor materials. The phosphor is part of the colour conversion element (CCE), which typically consists of phosphor particles embedded in a silicone matrix. As shown in [17], the white light quality critically depends on the arrangement of the CCE within the LED package. Moreover, the optical properties of the materials constituting the CCE and their temperature dependency, have a huge impact on the constancy of the chromaticity coordinates [18][19]. Furthermore, phosphors generally face the problem of decreasing luminescence intensity with increasing temperature. Consequently, the CCE is a distributed source of heat in the LED module, non-linearly dependent on temperature and light flux. The limited quantum efficiency and the accompanying Stokes-shift produce heat. Due to the rather low thermal conductivity of the silicone matrix, temperatures much higher than the LED junction temperature occur in the CCE.

The presented LED-model has successfully been used to investigate LED modules with attached CCE [20]. Optical ray tracing simulations provide a $3 \mathrm{D}$ map of the absorption of the blue light, the total blue light power is provided by the LED-model. The conversion of absorbed blue photons into emitted white photons is described by the temperature dependent quantum efficiency $Q E$ of the phosphor particles. This nonlinear efficiency number together with the Stokes shift (wavelength dependent light power) is responsible for the localised heat generation in the CCE.

Figure 9 shows a good correspondence between FEM simulation and IR thermography.

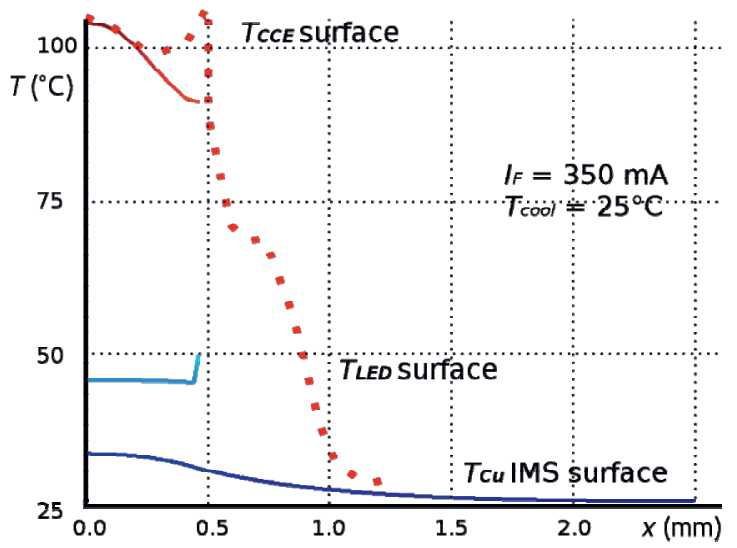

Fig. 9. Comparison of FEM simulation results for the LED module with CCE (solid lines) and results from emission-corrected IR-thermography (red dots: Infratec IR8300).

\section{Conclusions}

We have developed a rather complete model for phosphor converted high power LED modules. The parameterisation and verification of the LED-module is accomplished by a combined electrical-thermal-optical procedure. For a given LED forward current the temperature distribution throughout the module and the emitted light power are simulated, additionally allowing to determine the quality of the emitted light (e.g. colour constancy, chromaticity, colour rendering index, colour temperature). The presented model allows a systematic approach to optimise the performance of phosphor converted LED modules. Static and transient simulations can be used to evaluate the effect of varying materials parameters with their temperature dependencies, and excitation patterns on the complete LED-CCE-system, with respect to the emitted white light quality and stability, and lifetime aspects resulting from temperature. Several resulting aspects have been published by the authors, recently [17][18][19][20].

\section{Acknowledgement}

The LED samples have been prepared and kindly provided by Tridonic $\mathrm{GmbH}$, Jennersdorf.

The Center for Integrated Sensor Systems gratefully acknowledges partial financial support by the European Regional Development Fund (ERDF) and the province of Lower Austria (LNÖ). 


\section{References}

[1] E. F. Schubert, J. K. Kim, Solid-Light Sources Getting Smart, Science 308 (2005).

[2] J. K. Kim, E. F. Schubert, Transcending the replacement paradigm of solid-state lighting, Opt. Express 2008 (16) (2008).

[3] C. Sommer, P. Hartmann, P. Pachler, H. Hoschopf, F. P. Wenzl, White light quality of phosphor converted light-emitting diodes: A phosphor materials perspective of view, J. Alloy Comp. 520 (2012).

[4] D. S. Kim, B. Han, Effect of junction temperature on heat dissipation of high power light emitting diodes, J. Appl. Phys. 119 (2016).

[5] Y. Xi and E. F. Schubert, Junction-temperature measurement in $\mathrm{GaN}$ ultraviolet light-emitting diodes using diode forward voltage method, Appl. Phys. Lett. 85 (2004).

[6] A. Keppens, W. R. Ryckaert, G. Deconinck, P. Hanselaer, High power light-emitting diode junction temperature determination from currentvoltage characteristics, J. Appl. Phys. 104 (2008).

[7] X. Guo, E.F.Schubert, Current crowding in $\mathrm{GaN} / \mathrm{InGaN}$ light emitting diodes on insulating substrates, J. Appl. Phys 90(8) (2001)

[8] P. Fulmek, J. Nicolics, F. P. Wenzl, W. Nemitz, S. Schweitzer, P. Hartmann, Influence of the Electrode Structure on the Thermal Performance of High Power LEDs, Conf. Proc. 38th Int. Spring Seminar on Electronics Technology (2015).

[9] P. Fulmek, P. Haumer, F. P. Wenzl, W. Nemitz, J. Nicolics, Time dependent and temperature dependent properties of the forward voltage characteristic of InGaN high power LEDs, AIP Advances 7, 035206 (2017); doi: 10.1063/1.4978265 .

[10] Zi-Quan Guo, Tien-Mo Shih, Zhang-Bao Peng, Hai-Hua Qiu, Yi-Jun Lu, Yu-Lin Gao, Li-Hong Zhu, Jiang-Hui Zheng, Zhong Chen, On a relationship among optical power, current density, and junction temperature for InGaN-based lightemitting diodes, AIP Advances 7, 015307 (2017)

[11] P. Fulmek, C. Sommer, P Hartmann, P. Pachler, H. Hoschopf, G. Langer, J. Nicolics, F. P. Wenzl, On the Thermal Load of the Color-Conversion Elements in Phosphor-Based White LightEmitting Diodes, Adv. Optical Mater. 2013 (1) (2013)
[12] M. Meneghini, M. D. Lago, N. Trivellin, G. Meneghesso, E. Zanoni, Thermally Activated Degradation of Remote Phosphors for Application in LED Lighting, IEEE Trans. Device Mater. Rel. 13(1) (2013).

[13] P. Fulmek, C. Sommer, P Hartmann, P. Pachler, H. Hoschopf, G. Langer, J. Nicolics, F. P. Wenzl, On the Thermal Load of the Color-Conversion Elements in Phosphor-Based White LightEmitting Diodes, Adv. Optical Mater. 2013 (1) (2013)

[14] Cree-Inc., Data sheet: CPR3DX, Rev. B, (2015, accessed September, 2016), http://www.cree.com

[15] C. Geuzaine and J. F. Remacle. Gmsh: A threedimensional finite element mesh generator with built-in pre- and post-processing facilities. International J. Numerical Methods in Engineering 79 (11) (2009).

[16] P. Dular, C. Geuzaine, GetDP reference manual: the documentation for GetDP, a general environment for the treatment of discrete problems, http://getdp.info

[17] W. Nemitz, F.-P. Wenzl, S. Schweitzer, C. Sommer, P.Hartmann, P. Fulmek, J. Nicolics, A Self-Compensation Approach for Maintaining the Chromaticity Coordinates of Phosphor Converted LEDs Upon Temperature Variations, LED professional LpR 49 (2015)

[18] P. Fulmek, J. Nicolics, W. Nemitz, S. Schweitzer, C. Sommer, P.Hartmann, F. Schrank, F.-P. Wenzl, The impact of the thermal conductivities of the color conversion elements of phosphor converted LEDs under different current driving schemes, J. Luminescence 169 (2016).

[19] F. P. Wenzl, P. Fulmek, C. Sommer, S. Schweitzer, W. Nemitz, P. Hartmann, P. Pachler, H. Hoschopf, F. Schrank, G. Langer, J. Nicolics, Impact of extinction coefficient of phosphor on thermal load of color conversion elements of phosphor converted LEDs, J. Rare Earths 32(3) (2014)

[20] W. Nemitz, F.-P. Wenzl, S. Schweitzer, C. Sommer, P.Hartmann, P. Fulmek, J. Nicolics, Iterative Optical and Thermal Simulation Method for Proper Simulation of PC LEDs, LED professional LpR 52 (2015) 